\title{
BLOGS BRASILEIROS SOBRE SUICÍDIO
}

BRAZILIAN BLOGS ABOUT SUICIDE

Recibido: 14 de junio de 2018 | Aceptado: 22 de enero de 2019

\section{NADJA CRISTIANE LAPPANN BOTTI, , CAMILA CORRÊA MATIAS PEREIRA 2}

1. UNIVERSIDAdE FEDERAL dE SÃO JOÃO DEL REI, Divinópolis, Minas Gerais, Brasil

2. UNIVERSIDADE DE SÃO PAULO, São Paulo, Brasil

\begin{abstract}
Introduction: Suicidal behavior is an internet wide spread complex topic. Some websites support it; others are against it. Objective: To analyze the content of Brazilian blogs about suicide. Method: This is a descriptiveexploratory study. Blogs against and in favor of suicide were collected through Google. The criteria for the search included Brazilian blogs with more than one post about suicide, those with frequent posts about suicide in a year and blogs with posts until December 2014. Computer-mediated discourse analysis was used to analyze the sample. Results: Five blogs supporting suicide and five against were identified with features related to the levels of computer-mediated discourse analysis. Blogs against suicide present higher time of activity, higher number of messages, higher diversity regarding social networks connectivity, higher visits. They also include profile descriptions and use of real names in comparison with blogs supporting suicide. Conclusion: Internet holds relevant content that allows investigating risk factors and protection against suicide as well as the development of online prevention strategies.
\end{abstract}

KEY WORDS: Suicide, internet, mental health.

\section{CÓMO CITAR / HOW TO CITE}

Lappann Botti, N. C., \& Matias Pereira, C. C. (2019). Blogs brasileiros sobre suicídio. Salud \& Sociedad, 10(1), 10-19. doi: $10.22199 / S 07187475.2019 .0001 .00001$ 


\section{INTRODUÇÃO}

A Internet possibilitou a criação de novas comunidades representada por grupos de indivíduos online e também de novos modos de intervenção em saúde como grupos de suporte, consulta online e informações (Kirmayer, Raikher \& Rahimi, 2013). Neste sentido, a comunicação mediada por computador possibilita novo contexto comunicacional através de ferramentas que proporcionam a interação virtual em redes digitais como comunidades, fóruns e blogs (Recuero, 2012). Assim, a Internet caracteriza-se também como ferramenta social, principalmente pelo uso conversacional, sendo fundamental para a compreensão da sociedade contemporânea (Recuero, 2012).

$\mathrm{Na}$ sociedade atual as redes sociais digitais têm sido utilizadas na área da saúde para difusão de conhecimento ao possibilitar publicação e compartilhamento rápido de informações, associadas ao próprio ambiente virtual que produz maior interação (Lima et al., 2015). Estudo Brasileiro visando entender o perfil do usuário e as tendências de busca por informações de saúde na Internet aponta que a rede digital se tem mostrado fonte de informação relevante $e$ que a certificação de sites é uma estratégia a ser considerada na perspectiva de melhoria da qualidade das informações e promoção da saúde pública (Moretti, Oliveira \& Silva, 2012).

Como observado, o advento das redes digitais é responsável pela difusão de diversos assuntos, entre eles, 0 do comportamento suicida. Estudo internacional de revisão mostra que as pessoas usam a Internet na busca de informações sobre suicídio e/ou problemas relacionados com 0 suicídio. No entanto, ainda é incerto o nexo de causalidade entre o uso da Internet $e$ pensamento ou comportamento suicida (Mok, Jorm \& Pirkis, 2015). A busca de informações na Internet sobre o suicídio, apresenta duplo caráter uma vez que possibilita a realização de pacto suicida online e/ou informação sobre métodos, (podendo influenciar pessoas vulneráveis), mas também permite a avaliação da prevenção no contexto online (Ozawa, 2010; Chang, Kwok, Cheng, Yip \& Chen, 2015).

Observa-se que ambas as influências ocorrem em consequências dos diferentes contextos sociais e de vulnerabilidade (Niezen, 2013).

De acordo com o "Mapa da Violência", criado pelo Ministério da Saúde, o número de suicídios entre crianças e adolescentes de 10 a 14 anos no período de 2002 a 2012, aumentou $40 \%$. A prevalência do suicídio em crianças e adolescentes no Brasil aumentou de 0,9 a 1,1 mortes por 100.000 crianças e adolescentes no período de 2000 a 2010 (Waiselfisz, 2015; Sousa, 2017).

No Brasil, os dados do Sistema de Informações de Mortalidade (SIM) para o período de 1996 e 2015 mostram um total de 172.051 suicídios e dentre estes, 52.388 casos são de jovens entre 15 e 29 anos. Quanto à mortalidade geral, as lesões autoprovocadas intencionalmente são representadas por $0,8 \%$ em todas as idades e 3,6\% para a faixa etária de 15 a 29 anos, reafirmando a vulnerabilidade crescente do suicídio entre adolescentes e jovens e a necessidade de discussão do tema no país (Ribeiro \& Moreira, 2018).

A Internet é importante ferramenta para divulgação de informações que muitas vezes pode ser incorreta, questionável ou fraudulenta e assim, torna-se importante que os profissionais de Enfermagem utilizem a Web como aliada, atuando no compartilhamento de conteúdo em saúde, bem como na identificação dos possíveis fatores de risco e de proteção dos comportamentos suicidas online, afim de detectar populações vulneráveis para a prevenção do suicídio.

Devido ao crescente aumento do uso da Internet e da sua participação ativa na vida da maioria dos jovens, alguns estudos tem 
sido realizados no Brasil afim de utilizar o meio online como uma extensão do cuidado e investigação, como é o caso do estudo de Almeida \& Guimarães (2015) no trabalho sobre blogs "pró-ana", ligados à anorexia no sexo masculino e Rondina, Moura \& Carvalho (2016), investigando o Bullying online (Cyberbullying), no ambiente virtual.

Considerando que as redes sociais digitais têm sido utilizadas na busca de informações sobre suicídio associado ao caráter pró-suicida ou preventivo que a Internet pode ter, torna-se importante que os profissionais de Enfermagem reflitam sobre este problema a fim de atuarem na prevenção do suicídio por meio da identificação de fatores de risco e de proteção dos comportamentos suicidas online. Portanto, o objetivo deste trabalho foi analisar o conteúdo dos blogs Brasileiros sobre suicídio na Internet.

\section{MÉTODO}

Estudo descritivo exploratório realizado em blogs brasileiros localizados a partir da busca no Google com as palavras blog pró-suicídio, blogs suicídio, blog suicida e blogspot suicídio. Os critérios de inclusão foram: blogs Brasileiros com mais de uma postagem sobre suicídio, blogs com frequência de postagens no ano sobre suicídio durante 0 período de atividade e blogs com postagens até dezembro de 2014. A busca foi realizada no mês de Maio de 2015 e foram identificados 17 blogs e excluídos sete blogs de acordo com os critérios de inclusão, restando 10 blogs para a análise.

Para coleta de dados foi elaborado um instrumento para a construção do banco de dados contendo informações sobre o texto e data das postagens, descrições, nomes e imagens do perfil, 0 número de visualizações, conexões com outras redes sociais, o número de seguidores, enquete, canais de comunicação utilizados e layout do blog. A coleta foi realizada de abril a julho de 2015.
Realizado estudo qualitativo sendo utilizado para análise dos dados o método da Análise de Discurso Mediado por Computador (CMDA), idealizado por Herring, que investiga as formas de comunicação online produzidas através da transmissão de mensagens na Internet. Este referencial analisa cinco níveis da linguagem online: estrutura, significado, interação, comportamento social e comunicação multimodal (Herring, 2012).

Do mesmo modo que acontece no mundo real, ao se investigar as informações do ambiente online também é preciso observar questões éticas. Dessa forma, esta pesquisa foi aprovada pelo Comitê de Ética em Pesquisa da Universidade Federal de São João Del Rei (CAAE: 41774815.2.0000.5545).

\section{RESULTADOS}

Dentre os 10 blogs analisados, cinco apresentam conteúdo pró-suicidas (blogs pró-suicidas) e cinco tem conteúdo preventivo do comportamento suicida (blogs preventivos), apresentados na tabela a seguir (Tabela 1).

Os resultados da Análise de Discurso Mediado por Computador dos blogs sobre suicídio encontram-se apresentados no quadro 1. 
TABELA 1.

Caracterização do conteúdo dos blogs sobre suicídio (2008-2014), Brasil, 2015.

\begin{tabular}{cccccc}
\hline Conteúdo do blog & Blog & Data do primeiro post & Data do último post & \multicolumn{2}{c}{ Total de posts } \\
f & 13/10/2009 & $04 / 08 / 2011$ & 21 & 1,67 \\
\hline \multirow{3}{*}{ Pró-suicida } & 1 & $09 / 02 / 2012$ & $17 / 02 / 2012$ & 25 & 2,00 \\
& 2 & $01 / 09 / 2012$ & $01 / 09 / 2012$ & 4 & 0,31 \\
& 3 & $15 / 03 / 2013$ & $13 / 06 / 2013$ & 39 & 3,10 \\
& 4 & $09 / 03 / 2014$ & $21 / 10 / 2014$ & 40 & 3,18 \\
Preventivo & 5 & $31 / 07 / 2008$ & $04 / 10 / 2013$ & 367 & 29,22 \\
& 6 & $08 / 07 / 2009$ & $25 / 10 / 2014$ & 439 & 34,96 \\
& 7 & $08 / 05 / 2010$ & $27 / 07 / 2011$ & 52 & 4,14 \\
& 8 & $10 / 10 / 2010$ & $11 / 10 / 2014$ & 159 & 12,66 \\
& 9 & $04 / 05 / 2011$ & $08 / 11 / 2014$ & 110 & 8,76 \\
\hline
\end{tabular}

QUADRO 1.

Análise de Discurso Mediado por Computador dos blogs sobre suicídio (2008-2014), Brasil, 2015.

\section{Estrutura dos blogs}

Os blogs pró-suicidas apresentam: tempo médio de 203 dias ( 1 dia o menor tempo e 666 dias o maior tempo); número médio de 25,8 posts entre 2008 e 2014 (2014 com maior número de posts (40 posts/ano) e 2011 com menor número de posts (2 posts)) e $40 \%$ apresentam descrição do perfil

Os blogs preventivos apresentam: tempo médio dos blogs de 1403 dias (445 dias o menor tempo e 1935 dias o maior tempo); número médio de 225,4 posts entre 2008 e 2014 (2010 com maior número de posts (294 posts) e 2008 com menor número de posts (67 posts)) e 60\% apresentam descrição do perfil

Significado dos blogs

Os blogs pró-suicidas apresentam: pseudônimo com conteúdo pró-suicida (4 blogs) e apelido ( 1 blog); perfil com conteúdo de ideia de morte ( 2 blogs), sem descrição ( 2 blogs), ideia de pessimismo ( 1 blog); imagem do perfil (5 blogs) com desenho de violência ou pueril (colorido), fotografia mórbida ou pueril (preto/branco)

Os blogs preventivos apresentam: pseudônimo com nome próprio (3 blogs), com conteúdo mórbido (1 blog) e sigla (1 blog); perfil com conteúdo sem descrição (2 blogs), ideia científica (2 blogs), ironia (1 blog); imagem do perfil (4 blogs) com fotografia de retrato (colorida)

Interação dos blogs

Os blogs pró-suicidas apresentam: conexão com redes sociais de Conta Google (5) e Orkut (1); número de visualizações (2 blogs) [4619 e 2821 visualizações, respectivamente] e número de seguidores (2 blogs) [15 e 4 membros seguidores, respectivamente]

Os blogs preventivos apresentam conexão dos blogs com redes sociais de Conta Google (5), blogs de autoajuda (2), Twitter (2), Facebook (1), Orkut (1) e Youtube (1); número de visualizações (3 blogs) [92358, 19664 e 590,37 visualizações, respectivamente] e número de seguidores (5 blogs) [232, 63, 54, 36 e 13 membros seguidores, respectivamente]

\section{Comportamento social dos blogs}

A participação nos blogs pró-suicida e preventivo ocorre por meio de amigos, membros, colaboradores, seguidores e contato; as mensagens são assíncronas e transmitidas através de área de busca, arquivo, comentários, recomendações do blog, blogs amigos, lista de blogs e enquete; os canais de comunicação são textuais e imagens e as autorias das mensagens são do autor do blog e dos comentadores

\section{Comunicação multimodal dos blogs}

O layout dos blogs pró-suicida apresenta, em ordem de maior frequência, os recursos de mídia como foto, vídeo, música, desenho, quadro e animação

O layout dos blogs preventivos apresenta, em ordem de maior frequência, os recursos de mídia como foto, desenho, propaganda, pintura, vídeo, logos e imagens de notícia 


\section{DISCUSSÃO}

O primeiro blog com conteúdo sobre suicídio encontrado refere-se à publicação de um post em julho de 2008. Ressalta-se que foi exatamente dois anos após o lançamento das Diretrizes Brasileiras para um Plano Nacional de Prevenção do Suicídio (Ministério da Saúde, 2006).

A Internet possibilita que as pessoas tenham liberdade em criar um perfil não necessariamente fiel às suas características permitindo se apresentar nas redes sociais de acordo com a impressão que desejam transmitir. A partir da criação de um perfil, inicia-se a interação e são estabelecidas as conexões entre as pessoas (Herring, 2012). Observa-se que os autores dos blogs prósuicidas, na maioria, não apresentam descrição do perfil, mas exibem imagem do perfil que em geral são desenhos ou fotografias de caráter mórbido; publicam com pseudônimo com conteúdo pró-suicida e os perfis que são apresentados, mostram conteúdo com ideia de morte. Por outro lado, os blogs com conteúdo preventivo do comportamento suicida apresentam pseudônimo com nome próprio e apesar de apresentarem imagem do perfil, em geral de fotografia de retrato, não apresentam sua descrição do perfil.

As mudanças provocadas pela Internet 2.0 que lideram a geração digital englobam novas plataformas de comunicação interativa possibilitando as pessoas capacidade inédita para compartilhar experiências, somar vontades e agrupar-se para iniciar ações coordenadas. Deste modo, as ferramentas da Web 2.0 potencializam a ação coletiva no mundo real (Van Peborgh, 2010). Neste contexto, ressalta-se o conceito de capital social como valor para mensurar a qualidade dos laços em uma rede, onde a reciprocidade, confiança e consenso são aspectos da rede que englobam seus membros. 0 capital social reflete no espaço coletivo do grupo como valor constituído de acordo com as interações entre os atores sociais. Assim, o usuário não é simples consumidor de informação, mas alguém que começa a produzir conteúdo com possiblidade de várias alterações (Recuero, 2012).

Neste aspecto torna-se importante observar que os blogs preventivos apresentaram maior tempo médio de atividade (1403 dias) e número médio de postagens de 225,4 entre os anos de 2008 a 2014 quando comparados com os blogs prósuicidas, que mostram tempo médio de 203 dias e número médio de 25,8 postagens no período de 2008 a 2014. Esta diferença pode ser entendida pela própria característica do blog pró-suicida como diário eletrônico ou virtual. Os blogs são caracterizados como websites para publicação de informações periódicas, projetados originalmente para uso pessoal, caracterizando-os como diários pessoais online, em virtude de usuários utilizarem esse ambiente para narrar 0 cotidiano (Alao, Soderberg, Pohl \& Alao, 2006).

Os blogs preventivos apresentam maior tempo médio e maior diversidade de conexão com redes sociais como: Conta Google, blogs de autoajuda, Twitter, Facebook e Youtube e número de visualizações e seguidores quando comparados com os blogs pró-suicidas. A saúde pública também tem utilizado as redes sociais virtuais como célere meio de informação e capacitação sobre questões de saúde, além de mobilização social para parcerias e ações de intervenção em saúde (Antunes, Silva, Guimarães \& Rabaço, 2014). Importante ressaltar a diversidade de conexões encontradas nos blogs preventivos. Os blogs são mais que simples interfaces facilitadas para a publicação individual, pelo contrário, são espaços coletivos de interação (Yang \& Liu, 2009). Este aspecto evidencia a importância da Internet como ambiente colaborativo e a possiblidade da interação entre vários usuários na prevenção do suicídio.

Os blogs são considerados ambientes informacionais digitais colaborativos por 
permitirem a colaboração de vários usuários na inserção dos conteúdos e por isso, são considerados como novas ferramentas de comunicação e colaboração pessoal e de grupo, sendo o "blogar" (blogging) uma nova forma de publicação (Alao, et al., 2006). Dessa forma, o uso dos blogs adquire importância na sociedade atual para a prevenção do suicídio, especialmente, por publicarem informações, contatos e sites de ajuda para pessoas vulneráveis.

Os blogs preventivos apresentam maior descrição do perfil que os blogs pró-suicidas. Achado importante que pode favorecer a prevenção do suicídio pois ao ter a descrição do perfil, estes blogs podem ajudar pessoas com problemas de confiança, ideações paranoides ou desilusões, favorecendo a segurança, o que não ocorrem, em geral, quando o usuário é anônimo (Aderet, 2009). Os blogs preventivos também apresentam, em sua maioria, nome próprio confirmando que 0 anonimato na Internet impede a verificação da autenticidade das informações de um site tornando, neste caso os blogs preventivos, como mais confiáveis (Gilat \& Shahar, 2007). A Internet também atua como estratégia de prevenção do suicídio ao possibilitar a divulgação profissional suporte emocional, principalmente através de chats, grupos e fóruns, que podem auxiliar no contato de procura de ajuda (Luxton, June \& Fairall, 2012).

Os blogs pró-suicidas apresentam em sua maioria pseudônimo com conteúdo prósuicida e imagens mórbidas ou de violência em preto/branco. Este achado pode se justificar pelo fato de que 0 anonimato na Internet facilita a comunicação de ideias suicidas com a publicação de pensamentos de morte, falta de esperança e/ou métodos de perpetração do suicídio (Barak \& Miron, 2005). Assim, entre os desafios para 0 estudo do comportamento suicida na Internet encontra-se que a maioria das pessoas deseja ficar anônima, muitas vezes a fim de evitar estigmas ou intervenções preventivas, apesar de comunicarem suas ideias $e$ intenções suicidas (Mishara \& Weisstub, 2007). Os blogs pró-suicidas também apresentam, em sua maioria, perfis com ideias de morte. Este dado pode ser explicado, uma vez que obter informação pró-suicida na Internet, geralmente refere-se a busca de informações acerca de métodos para cometê-lo (Robertson, Skegg, Poore, Williams \& Taylor, 2012).

A conexão dos blogs é mais diversificada nos blogs preventivos quando comparado com os blogs pró-suicidas. Os fóruns e chats existentes nas redes sociais podem ser considerados como risco para grupos vulneráveis uma vez que as interações online podem encorajar, idolatrar pessoas que suicidaram e facilitar pactos suicidas influenciando a decisão de morrer por suicídio (Robertson, et al., 2012). Ao mesmo tempo, o vínculo com grupos de apoio online pode facilitar conexões, compartilhamento de experiências e programas de prevenção. Neste contexto, o Youtube e o Facebook oferecem serviços de ajuda e contato de programas de prevenção do suicídio (Robertson, et al., 2012).

Os blogs pró-suicidas utilizam menos ferramentas de visualizações do blog e de contagem do número de seguidores, enquanto os blogs preventivos utilizam mais tais recursos. Entre as implicações da Internet sobre as pessoas que estão acessando ativamente informações sobre 0 suicídio encontra-se o "efeito de contágio", presente nas diversas formas de redes sociais virtuais. A ideia de contágio vem pelo qual um determinado suicídio facilita a ocorrência de outros suicídios, a despeito do conhecimento direto ou indireto do suicídio prévio em um determinado contexto em detrimento da comunicação (Fu, Cheng, Wong \& Yi, 2013). O que afirma a importância da conexão tanto nos blogs prósuicidas quanto nos preventivos no aspecto dos seus objetivos.

Pouca diferenciação foi encontrada nos blogs pró-suicidas e preventivos quanto a 
participação, a assincronicidade das mensagens, os canais de comunicação mais comuns (texto e imagem) e as autorias das mensagens (autor do blog e dos comentadores). A diferença encontra-se no propósito da comuni<cação (comportamento pró-suicida ou prevenção do comportamento suicida) e que os blogs preventivos possuem mais fontes de dados do que os pró-suicidas. A comunicação online, através de mensagens com conteúdos de encorajamento do suicídio pode favorecer 0 suicídio, porém, também se encontram na Internet, suporte emocional de serviços ou grupos de ajuda online que podem ser eficientes na prevenção do suicídio (Mishara \& Weisstub, 2007).

$\mathrm{Na}$ Internet existem sites que apresentam informações de encorajamento do suicídio com descrição detalhada de métodos e mensagens de "encorajadores de suicídio" que atuam como "predadores de suicidas" ao procurarem pessoas que postam mensagens sugerindo comportamento suicida, aproveitam para incitar ao disponibilizar orientações de como cometê-lo (Gilat \& Shahar, 2007). Neste aspecto, problematizase a questão da influência da Internet sobre - comportamento suicida gerando a pergunta: o suicídio pode ocorrer em função do encorajamento ao ato? A formação de grupos identitários pode apresentar comunicação online de valores heroicos do suicídio e assim, influenciar atitudes de autodestruição, possivelmente em benefício da inclusão num grupo. Além disso, alguns sites da Internet incentivam pessoas a divulgarem informações e métodos de suicídio (Niezen, 2013). Estudo sobre a Internet e o suicídio enfatiza que grupos de suporte online favorecem a prevenção do suicídio a partir do encorajamento de busca de ajuda (Gilat \& Shahar, 2007). Neste sentido, se existem sites promovendo a ideia do suicídio e encorajando seus participantes em atos autolesivos, a melhor estratégia seria a criação de sites de aconselhamento e/ou suporte de promoção de valores positivos em favor da vida (Niezen, 2013).
Em ambos os blogs do estudo verifica-se que os recursos de mídia mais utilizados se referem às fotos e em menor frequência os vídeos, músicas, desenhos, quadros, animações, propaganda, pintura, logos e imagens de notícia. A questão do uso de recursos de mídia torna-se importante, principalmente porque a maior parcela dos usuários da Web é jovem. Sendo assim, a comunicação virtual de material sobre 0 suicídio pode encorajar jovens vulneráveis ao suicídio por serem as que apresentam maior chance de encontrar conteúdo associado ao suicídio na Internet (Luxton, et al., 2012).

\section{CONCLUSÃO}

Os achados afirmam que existe importante conteúdo sobre suicídio na Internet possibilitando a investigação sobre os fatores de risco e de proteção. Também se observa as principais características dos blogs pró-suicidas e preventivos em diversos níveis de análise possibilitando, principalmente a identificação de um perfil a ser buscado em estratégias de prevenção do suicídio nas comunicações online.

Os blogs pró-suicidas apontam para 0 desafio do cuidado às pessoas que apresentam comunicação online de risco de suicídio e não buscam serviços de saúde, assim destaca-se a importância do conhecimento da realidade virtual da comunicação sobre suicídio para evitar que informações pró-suicidas que atinjam 0 público vulnerável sejam maiores que as preventivas.

Sendo a Internet uma ferramenta de uso crescente e cada vez mais presente na vida das pessoas, os enfermeiros e demais profissionais de saúde, precisam de treinamento sobre a identificação, abordagem e ajuda às pessoas com comportamento suicida online, devido a vulnerabilidade que este grupo apresenta aos blogs pró-suicidas. Neste sentido tornase importante garantir que a Web seja um ambiente seguro com informações de 
valorização da vida, prevenção do suicídio e divulgação de serviços de ajuda online e offline.

O estudo apresenta limitações quanto à utilização específica de blogs brasileiros gratuitos sendo que ainda na Internet encontram-se também sites, chats, fóruns e blogs hospedados em plataformas pagas com conteúdo sobre o suicídio. Ressalta-se que existem poucos estudos sobre prevenção online do suicídio e que há a necessidade da construção de políticas públicas de saúde específicas da comunicação na Web.

\section{REFERÊNCIAS}

Aderet, A. (2009). Alert: The Dark Side of Chats-Internet without Boundaries. The Israel Journal of Psychiatry and Related Sciences, 46(3), 162-166.

Alao, A.O., Soderberg, M., Pohl, E.L. \& Alao, A.L. (2006). Cybersuicide: review of the role of the internet on suicide. Cyberpsychology Behaviour, 9(4), 489493.

Almeida, T. C.,\& Guimarães, C. F. (2015). Os blogs pró-Ana e a experiência da anorexia no sexo masculino. Saúde e Sociedade, 24(3), 1076-1088.

Antunes, M.N., Silva, C.H., Guimarães, M.C.S. \& Rabaço, M.H.L. (2014). Monitoramento de informação em mídias sociais: 0 e-Monitor Dengue. Transinformação, 26(1), 9-18.

Barak, A. \& Miron, O. (2005). Writing characteristics of suicidal people on the Internet: a psychological investigation of emerging social environments. Suicide and Life-Threatening Behavior, 35(5), 507-524.

Chang, S. S., Kwok, S. S., Cheng, Q., Yip, P. \& Chen, Y. Y. (2015). The association of trends in charcoal-burning suicide with Google search and newspaper reporting in Taiwan: a time series analysis. Social Psychiatry and Psychiatric Epidemiology, 50(9), 1451-1461.

Fu, K.W., Cheng, Q., Wong, P.W.C. \& Yi, P.S.F. (2013). Responses to a self- presented suicide attempt in social media. Crisis: The Journal of Crisis Intervention and Suicide Prevention, 34(6), 406-412.

Gilat, I. \& Shahar, G. (2007). Emotional first aid for a suicide crisis: comparison between Telephonic hotline and internet. Psychiatry, 70(1), 12-8.

Herring, S.C. (2012). Discourse in Web 2.0: Familiar, reconfigured, and emergent. In D. Tannen \& A. M. Tester (Eds.), Georgetown University Round Table on Languages and Linguistics 2011: Discourse 2.0: Language and new media, 1-25.

Kirmayer, L.J., Raikhel, E. \& Rahimi, S. (2013). Cultures of the Internet: Identity, community and mental health. Transcultural Psychiatry, 50(2), 165191.

Lima, S.G.P., Guimarães, K.M.F., Gerhardt, P.C., Cassiano, A.C.M., Vermelho, S.C.S.D. \& Bortolozzi, F. (2015). A utilização de redes sociais digitais na área da saúde: uma revisão sistemática. Saúde e Pesquisa, 8, 79-91.

Luxton, D.D., June, J.D. \& Fairall, J.M. (2012). Social Media and Suicide: A Public Health Perspective. American Journal of Public Health, 102(Suppl 2), S195-S200.

Ministério da Saúde. (2006). Portaria $n^{0}$ 1.876 de 14 de agosto de 2006. Diretrizes brasileiras para um plano nacional de prevenção do suicídio.

Mishara, B.L. \& Weisstub, D.N. (2007). Ethical, legal and practical issues in the control and regulation of suicide promotion and assistance over the Internet. Suicide and Life-Threatening Behavior, 37(1), 58-65.

Mok, K., Jorm, A.F. \& Pirkis, J. Suiciderelated Internet use: A review. Australian and New Zealand Journal of Psychiatry, 49(8), 697-705.

Moretti, F.A., Oliveira, V.E. \& Silva, E.M.K. (2012). Acesso a informações de saúde na internet: uma questão de saúde pública? Revista da Associação Médica Brasileira, 58(6), 650-658. 
Niezen, R. (2013). Internet suicide: Communities of affirmation and the lethality of communication. Transcultural Psychiatry, 50(2), 303-322.

Ozawa, D.S.C. (2010). Shared death: Self, sociality and Internet group suicide in Japan. Transcultural Psychiatry, 47(3), 392-418.

Recuero, R. (2012). A conversação em rede. Porto Alegre: Sulina.

Ribeiro, J.M. \& Moreira, M.R. (2018). Uma abordagem sobre 0 suicídio de adolescentes e jovens no Brasil. Ciência saúde coletiva [online], 23(9), 28212834.

Robertson, L., Skegg, K., Poore, M., Williams, S. \& Taylor, B. (2012). An adolescent suicide cluster and the possible role of electronic communication technology. Crisis, 33, 239-245.

Rondina, J. M., Moura, J. L. \& Carvalho, M.D. (2016). Cyberbullying: o complexo bullying da era digital. Revista de Saúde Digital e Tecnologias Educacionais, 1(1), 20-41.
Sousa, G. S. de, Santos, M. S. P. dos, Silva, A. T. P. da, Perrelli, J. G. A. \& Sougey, E. B. (2017). Revisão de literatura sobre suicídio na infância. Ciência \& Saúde Coletiva, 22(9), 3099-3110.

Van Peborgh, E. (2010). Odisea 2.0 - Las Marcas e lós Medios Sociales. Buenos Aires: La Crujía.

Yang, H.L. \& Liu, C.L. (2009) A new standard of on-line customer service process: Integrating language-action into blogs. Computer Standards \& Interfaces, 31(1), 227-245.

Todos los trabajos publicados en

Revista Salud \& Sociedad (ISSNe:0718-7475)

están sujetos a una licencia Creative Commons

Reconocimiento 4.0 Internacional

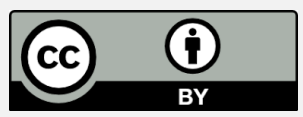




\section{RESUMO}

Introdução: 0 comportamento suicida é um assunto complexo e muito difundido na Internet, com conteúdo preventivo e pró-suicida, diretamente ligados à fatores de risco e de proteção do suicídio. Objetivo: Analisar o conteúdo dos blogs brasileiros sobre suicídio na Internet. Método: Estudo descritivo exploratório. Os blogs foram localizados na busca no Google com as palavras blog pró-suicídio, blog suicídio, blog suicida e blogspot suicídio. Critérios de inclusão: blogs Brasileiros com mais de uma postagem sobre suicídio, blogs com frequência de postagens no ano sobre suicídio durante o período de atividade e blogs com postagens até dezembro de 2014. A análise foi realizada através da Análise do Discurso Mediado pelo Computador. Resultados: Foram identificados cinco blogs com conteúdo pró-suicida e cinco preventivos apresentando características em relação aos níveis da Análise do Discurso Mediado pelo Computador. Os blogs preventivos apresentaram maior tempo médio de atividade, maior número médio de postagens, maior diversidade de conexão com redes sociais, visualizações, descrições do perfil e uso de nome próprio quando comparados aos blogs pró-suicidas. Conclusão: Existe importante conteúdo sobre suicídio na Internet que possibilita a investigação dos fatores de risco e de proteção do suicídio, bem como o desenvolvimento de estratégias de prevenção online.

PALAVRAS-CHAVE: Suicídio, Internet, Saúde Mental.

\section{RESUMEN}

Introducción: El comportamiento suicida es un tema complejo y muy difundido en Internet, con contenido preventivo y pro-suicida, directamente relacionados a factores de riesgo y de protección del suicidio. Objetivo: Analizar el contenido de los blogs brasileños sobre el suicidio en Internet. Método: Estudio descriptivo exploratorio. Los blogs se localizaron a partir de la búsqueda en Google bajo los descriptores blog pro-suicidio, blog suicidio, blog suicida y blogspot suicidio. Los criterios de inclusión fueron: blogs Brasileños con más de un post sobre suicidio, con frecuencia de posts sobre suicidio en el año durante el período de actividad y blogs con posts hasta diciembre de 2014. El análisis se realizo a través del método de Análisis del Discurso Mediado por el computador. Resultados: Se identificaron cinco blogs con contenido pro-suicida y cinco preventivos presentando características con relación a los niveles del Análisis del Discurso Mediado por el computador. Los blogs preventivos presentaron mayor tiempo medio de actividad, mayor número medio de mensajes, mayor diversidad de conexión con redes sociales, visualizaciones, descripciones del perfil y uso de nombre propio en comparación con los blogs pro-suicidas. Conclusión: Hay un importante contenido sobre el suicidio en Internet que permite la investigación de los factores de riesgo y de protección del suicidio, así como el desarrollo de estrategias de prevención en línea.

PALABRAS CLAVE: Suicidio, Internet, Salud Mental. 